Published in final edited form as:

Occup Environ Med. 2012 June ; 69(6): 437-443. doi:10.1136/oemed-2011-100119.

\title{
Basal ganglia intensity indices and diffusion weighted imaging in manganese-exposed welders
}

\author{
Susan R Criswell ${ }^{1,2}$, Joel S Perlmutter ${ }^{1,2,3,4,5,6}$, John L Huang ${ }^{1,2}$, Nima Golchin ${ }^{1}$, Hubert P \\ Flores $^{1}$, Angela Hobson ${ }^{1,2}$, Michael Aschner ${ }^{7}$, Keith M Erikson ${ }^{8}$, Harvey Checkoway $^{9}$, and \\ Brad A Racette ${ }^{1,2}$ \\ ${ }^{1}$ Department of Neurology, Washington University School of Medicine, St. Louis, Missouri, USA \\ ${ }^{2}$ American Parkinson Disease Association Advanced Center for Parkinson Research, St. Louis, \\ Missouri, USA \\ ${ }^{3}$ Department of Radiology, Washington University School of Medicine, St. Louis, Missouri, USA \\ ${ }^{4}$ Department of Anatomy and Neurobiology, Washington University School of Medicine, St. Louis, \\ Missouri, USA \\ ${ }^{5}$ Program in Occupational Therapy, Washington University School of Medicine, St. Louis, \\ Missouri, USA \\ ${ }^{6}$ Program in Physical Therapy, Washington University School of Medicine, St. Louis, Missouri, \\ USA \\ ${ }^{7}$ Department of Pediatrics, Vanderbilt University Medical Center, Nashville, Tennessee, USA \\ ${ }^{8}$ Department of Nutrition, University of North Carolina-Greensboro, Greensboro, North Carolina, \\ USA
}

${ }^{9}$ Department of Environmental and Occupational Health Sciences, University of Washington School of Public Health, Seattle, Washington, USA

\section{Abstract}

\footnotetext{
Correspondence to Dr Susan R Criswell, Department of Neurology, Washington University School of Medicine, 660 South Euclid Ave., Box 8111, St. Louis, MO 63110, USA; criswells@neuro.wustl.edu.

Contributors All authors of this paper meet the uniform requirements for authorship based on substantial contribution to: (1) conception and design or analysis and interpretation of data, (2) drafting the article or revising it critically for important intellectual content and (3) have given final approval of the version to be published. There is no one else who fulfils the criteria but has not been included as an author. Contributorship is as follows: (1) SRC: planning/recruiting, conducting imaging processing/analysis and reporting/critical revision of the work. (2) JSP: study planning, imaging processing/analysis and reporting/critical revision of the work. (3) JH: recruiting, conducting imaging processing/analysis and reporting/critical revision of the work. (4) NG: conducting imaging processing/analysis and reporting/critical revision of the work. (5) HPF: conducting imaging processing/analysis and reporting/critical revision of the work. (6) AH: recruiting, conducting exposure analysis and reporting/critical revision of the work. (7) MA: conducting serum manganese analysis and reporting/critical revision of the work. (8) KE: conducting serum manganese analysis and reporting/ critical revision of the work. (9) HC: study planning and design and reporting/critical revision of the work. (10) BAR: study planning and design/recruiting and reporting/critical revision of the work. The corresponding author SRC will serve as the guarantor of the work and accepts full responsibility for the work and/or the conduct of the study. She had access to the data and controlled the decision to publish.

Patient consent Obtained.

Ethics approval Ethics approval was provided by Washington University School of Medicine Human Research Protection Office. Provenance and peer review Not commissioned; externally peer reviewed.

Data sharing statement The authors have full access to all the data and have the right to publish any and all data separate and apart from any sponsor. Data have not been shared beyond the authors listed and are presented in aggregate to protect individual-subject confidentiality.
} 
Objectives-Manganese exposure leads to diffuse cerebral metal deposition with the highest concentration in the globus pallidus associated with increased T1-weighted MRI signal. T1 signal intensity in extra-pallidal basal ganglia (caudate and putamen) has not been studied in occupationally exposed workers. Diffusion weighted imaging is a non-invasive measure of neuronal damage and may provide a quantification of neurotoxicity associated with welding and manganese exposure. This study investigated extra-pallidal $\mathrm{T} 1$ basal ganglia signal intensity as a marker of manganese exposure and basal ganglia diffusion weighted imaging abnormalities as a potential marker of neurotoxicity.

Methods-A 3T MR case:control imaging study was performed on 18 welders and 18 age- and gender-matched controls. Basal ganglia regions of interest were identified for each subject. T1weighted intensity indices and apparent diffusion coefficients were generated for each region.

Results-All regional indices were higher in welders than controls ( $\mathrm{p} \unlhd$ 0.05). Combined basal ganglia $(\rho=0.610)$, caudate $(\rho=0.645)$, anterior $(\rho=0.595)$ and posterior putamen $(\rho=0.511)$ indices were more correlated with exposure than pallidal $(\rho=0.484)$ index. Welder apparent diffusion coefficient values were lower than controls for globus pallidus $(\mathrm{p}=0.03)$ and anterior putamen $(\mathrm{p}=0.004)$.

Conclusions-Welders demonstrated elevated T1 indices throughout the basal ganglia.

Combined basal ganglia, caudate and putamen indices were more correlated with exposure than pallidal index suggesting more inclusive basal ganglia sampling results in better exposure markers. Elevated indices were associated with diffusion weighted abnormalities in the pallidum and anterior putamen suggesting neurotoxicity in these regions.

\section{INTRODUCTION}

Over 1 million workers in the USA weld as part of their job duties. ${ }^{1}$ Exposure to welding fume and its constituents, particularly manganese $(\mathrm{Mn})$ may produce basal ganglia neurotoxicity resulting in clinical parkinsonism. ${ }^{2}$ Parkinsonism is a clinical syndrome characterised by tremor, rigidity, bradykinesia and postural instability. ${ }^{3}$ While an exposureresponse relationship between Mn or welding fume and parkinsonism has not yet been determined, high levels of Mn exposure can result in deposition in the brain ${ }^{4-6}$ with a characteristic increased signal on T1-weighted MR imaging in the globus pallidus. ${ }^{78}$ The intensity of the pallidal signal correlates with welding exposure ${ }^{9}$ and Mn blood levels ${ }^{10}$ but is less clearly linked with clinical symptomatology, including parkinsonism. In addition, the relationship between signal intensity and neuronal pathology is unknown. Potentially, a more sensitive T1-weighted marker could provide an improved marker of exposure and potentially an antecedent biomarker of Mn neurotoxicity prior to onset of clinical symptomatology. Such a biomarker could be a critical tool to investigate the neurotoxicity of metal exposures and permit screening of at-risk workers.

Patients with cirrhosis, at risk for Mn toxicity due to impaired Mn clearance, have extrapallidal T1 hyperintesities, including abnormal signal in caudate and putamen. ${ }^{1112}$ However, extra-pallidal deposition of Mn with subsequent T1 hyperintensities and their relationship with exposure have not been explored in workers with occupational exposures to Mn. Nonhuman primates demonstrate greatest Mn deposition in globus pallidus, but Mn also accumulates in caudate and putamen. ${ }^{4}$ Similarly, neuropathological examinations in humans with symptomatic clinical Mn toxicity reveal degeneration of the medial segment of the globus pallidus with less severe degeneration of the putamen and caudate. ${ }^{13}$ Additional evidence that welding affects non-pallidal basal ganglia structures comes from the observation that PET measured uptake of $\left[{ }^{18} \mathrm{~F}\right] \mathrm{FDOPA}$ is reduced in the caudate of asymptomatic welders. ${ }^{14}$ Together these findings suggest that extra-pallidal hyperintensities are likely present in occupationally exposed welders. Intensity measurement of these regions 
may prove to be improved markers of exposure and its relationship to clinical symptomatology.

Structural T1-weighted imaging is an established marker of Mn exposure but remains limited as it does not provide information about function or neuropathology. Diffusion weighted (DW) and diffusion tensor imaging (DTI) non-invasively measure the Brownian movement of water molecules (diffusion) in the brain. ${ }^{15}$ The movement of water is measured in terms of the apparent diffusion coefficient (ADC). Changes in the ADC represent disruption in the movement of water secondary to changes in neuronal architecture. ${ }^{15}$ Diffusion weighted imaging (DWI) can easily be acquired during standard brain MRI imaging and provides detailed information about neuronal structural integrity. Restricted diffusion, as measured by ADC, is associated with the accumulation of sodium and water within a neuron leading to cytotoxic edema and neuronal death. ${ }^{16}$ A previous case study using DWI reported restricted diffusion in the globus pallidus of a patient with Mn toxicity secondary to liver failure. ${ }^{17}$

Our primary hypothesis was that Mn exposure in welders would be associated with elevated T1 signal indices in globus pallidus, caudate, anterior and posterior putamen and the intensity of the signal in all four regions would directly correlate with welding exposure. Our secondary hypothesis was that the average DWI ADC values across these basal ganglia regions would be reduced in areas with elevated intensity indexes indicating local neuropathological changes. Demonstration of pallidal and extra-pallidal DW abnormalities in Mn-exposed welders that correlate with T1 markers of exposure could provide a critical link in understanding the relationship between the level of Mn exposure and the onset of clinical neurotoxicity.

\section{METHODS}

This study was approved by the Washington University School of Medicine Human Research Protection Office, and all subjects signed a written consent. Welder data are presented in aggregate to protect individual-subject confidentiality. Welders were recruited from two Midwestern shipyards and one fabrication company from April 2007 through November 2010. The welding processes most commonly used by these welders were flux core arc welding, shielded metal arc welding and gas metal arc welding mainly on carbon steel. ${ }^{14}$ For our power calculation, we assumed that exposed welders would have caudate and putamen indices at least 5 points (5\%) higher than unexposed controls. A sample size of 20 per group, anticipating a loss of two subjects per group, provided over $80 \%$ power to detect a 5\% difference between welders and normal subjects even using an a level of 0.01 (assuming there are multiple comparisons in the analysis). Healthy age- and gender-matched control subjects were recruited for a 1:1; case:control match with the welders who had completed scans. Control subjects were community volunteers who were referred from our extensive regional volunteer network and were individually aged matched for welders \pm 4 years. All subjects were evaluated by a Movement Disorders specialist blinded to exposure status with an examination that included a Unified Parkinson's Disease Rating Scale motor subsection 3 (UPDRS3), which includes objective rating of the cardinal features of parkinsonism-tremor, rigidity, bradykinesia and postural instability. ${ }^{18}$ Welders were excluded from participation if they had $<100 \mathrm{~h}$ of welding exposure or if they had comorbid neurological disease that affected the UPDRS3 rating. Control subjects were excluded if they had a total UPDRS3 score of $>3$ or an individual rest, postural or action tremor score of $>1$. All subjects were screened for prior drug (prescription and recreational) exposures and were excluded for neuroleptic or amphetamine use. Welding subjects completed a validated exposure questionnaire and provided blood samples for Mn levels. ${ }^{19}$ Self-reported welding days/week and hours/day per job were multiplied by the number of weeks held at that job. 
This resulted in the number of hours a participant welded per job. Cumulative exposure hours were calculated by summing the per job welding hours. Blood Mn levels were analysed with graphite furnace atomic spectrometry (Varian AA240; Varian, Inc., Agilent Technologies, Santa Clara, CA USA) following published methods. ${ }^{20}$

\section{MRI studies}

Imaging was performed on a 3.0T Siemens Trio scanner (Erlangen, Germany). Structural anatomic scans included a T1-weighted sagittal, magnetisation-prepared rapid gradient echo (MPRAGE; repetition time $(\mathrm{TR})=2400 \mathrm{~ms}$; inversion time $(\mathrm{TI})=1000 \mathrm{~ms}$, echo time (TE) $=3.14 \mathrm{~ms}$, flip angle $=8^{\circ}, 0.9 \times 0.9 \times 0.9 \mathrm{~mm}$ voxels $)$ and a $\mathrm{T} 2$-weighted $(\mathrm{T} 2 \mathrm{~W})$ fast spin echo $(\mathrm{TR}=3200 \mathrm{~ms}, \mathrm{TE}=469 \mathrm{~ms}, 0.9 \times 0.9 \times 0.9 \mathrm{~mm})$.

To prevent any bias in analysis, a reviewer blinded to the clinical status of the subject outlined volumes of interest (VOIs), including the caudate, globus pallidus, anterior and posterior putamen and occipital regions on individual MR images. The intensity of the signal on the T1 weighted image in the VOI was compared by calculating an intensity index for each subject. The intensity index was defined as the ratio of T1 signal in the VOI to a white matter reference region. Regional indices were calculated from the T1 MPRAGE images as previously described for the pallidal index. ${ }^{21}$

Intensity index $=[($ Left VOI+Right VOI $) /($ Left white matter control region+Right white matter control region) $] \times 100$

Intensity indices were created for the globus pallidus, caudate, anterior putamen and posterior putamen using the same reference control regions. A combined basal ganglia intensity index was created by averaging the intensity indexes of all four VOIs.

Three sets of DTI images were acquired in 25 directions, at multiple b values, using an echo planar imaging sequence $(\mathrm{TR}=9200 \mathrm{~ms}, \mathrm{TE}=90 \mathrm{~ms}, 2.0 \mathrm{~mm}$ isotropic voxels, $\mathrm{b}$ values $=0$ $1400 \mathrm{~s} / \mathrm{mm}^{2}$ ). Diffusion analysis was performed using standard log-linear regression ${ }^{22}$ with software developed in the Neuroimaging Laboratory at Washington University as previously described. ${ }^{23}$

\section{Statistical analysis}

Age and UPDRS3 scores were compared between the welders and control groups using Mann-Whitney U tests. Within the welder cohort, relationships between Mn blood levels, welding exposure hours, regional indices and ADC values were examined with Spearman's correlations. A hierarchical linear regression model was used in a series of forced entry regression models to examine if adding additional intensity indices to the gold standard pallidal index would improve the ability to predict welding exposure by comparing the additional variance explained. Repeated measures generalised linear modelling (GLM) analysis was used to examine the effects of diagnostic group and region on regional intensity indexes and ADC values. In addition, the interaction between diagnostic group (betweensubjects variable) and region (within-subject variable) from the repeated measures GLM analysis was used to examine differences in the patterns of these variables across regions in the two groups. Post hoc Mann-Whitney U tests were performed to compare differences between groups for each region. The statistical software SPSS for Windows V.17.0 was used for data analysis.

\section{RESULTS}

We screened 193 subjects with a history of welding exposure to participate in this study. Of subjects contacted, 20 were unable to participate due to exclusionary criteria (imbedded 
metal, exclusionary medication, unable to fit in scanner), 20 refused to participate and the remainder were scanned or were willing to be scanned at a future date. The scanned subjects did not differ significantly from the entire cohort or those who were excluded based on age, gender, race, tobacco use or welding hours. Those scanned were younger than those who refused (mean age 41.0 and 54.6 years, respectively, $\mathrm{p}<0.01$ ) but did not differ significantly on gender, race, tobacco use or welding hours. Of the 20 welders originally scanned, two subjects' MRIs could not be analysed secondary to poor head position from scoliosis in one subject and excessive motion artefact in another. The remaining 18 welders with completed scans were compared with 18 age- and gender-matched control subjects. Demographic characteristics for the two diagnostic categories are shown in table 1 . Welders had median lifetime exposure of 9862 (IQR 2138.18-24 719.86) welding hours and average Mn levels two times the upper limits of normal $(24.15 \pm 17.6 \mu \mathrm{g} / 1$, normal values $<10.8 \mu \mathrm{g} / \mathrm{l})$. Welders demonstrated mildly elevated median UPDRS3 scores (6.25) despite no clinical symptoms and were different from the control group (table 1). UPDRS3 scores and blood Mn levels did not correlate with exposure hours or the age of the subject.

Repeated measures GLM analysis of the intensity indexes demonstrated a main effect of group, $\mathrm{F}(1,33)=13.76$, $\mathrm{p}=0.001$, and region, $\mathrm{F}(3,102)=894.96$, $\mathrm{p} \unlhd) .001$, indicating betweensubject differences between the two groups and within-subject differences between regions. There was also a strong interaction between region and group within the model, $\mathrm{F}(3,102)=6.16, \mathrm{p}=0.001$, indicating a different pattern of $\mathrm{T} 1$ intensity indices across regions in the diagnostic groups. Post hoc Mann-Whitney U tests revealed that intensity indices (left and right side averaged) were significantly higher in welders than controls for all regions (table 2). The greatest relative difference was present in the combined basal ganglia index, globus pallidus and posterior putamen.

Within the welder cohort, the combined global basal ganglia intensity index (Spearman's correlation, $\rho=0.610, p=0.007)$, caudate index $(\rho=0.645, p=0.004)$, anterior putamen $(\rho=0.595, p=0.009)$ and the posterior putamen index $(\rho=0.571, p=0.013)$ were all correlated with exposure hours. All four indices were more correlated with exposure than the pallidal index (Spearman's correlation $\rho=0.484, \mathrm{p}=0.042$ ). The exposure-imaging correlations are illustrated in figure 1 . None of the indexes were correlated with the age of either welder or control subjects. Welder blood Mn levels were inversely correlated with caudate index ( $\rho=$ $-0.0552, \mathrm{p}=0.012$ ) but none of the other intensity indices. UPDRS3 values were not correlated with intensity indices within the welder or control cohort.

The hierarchical linear regression analysis using individual intensity indices as predictors of welding exposure hours within the welder group is depicted in table 3 . Additional indices did improve the overall model but the caudate intensity index was the most predictive variable ( $\beta=0.485$ vs pallidal index, $\beta=0.012$ ) and provided the only significant $R^{2}$ change in the analysis. A separate linear regression analysis using the combined basal ganglia intensity index of welders revealed the combined index had an $R$ value of 0.489 and an $R^{2}$ value of 0.239 in predicting exposure hours.

Repeated measures GLM analysis of the regional ADC values demonstrated a main effect of region, $\mathrm{F}(3,32)=40.36, \mathrm{p} \unlhd .001$, and group, $\mathrm{F}(3,32)=8.43$, $\mathrm{p}=0.006$ ), for the model, but group and the interaction between region and group was not significant indicating the pattern of intensity indices across regions was the same in both groups (parallel). Given the significant differences in the intensity values and our primary hypothesis, we performed Mann-Whitney tests on the individual regions as described in table 4. ADC values were lower in welders compared to controls in the globus pallidus $(\mathrm{U}=93.0, \mathrm{p}=0.03)$ and anterior putamen $(\mathrm{U}=71.5, \mathrm{p}=0.004)$. The $\mathrm{ADC}$ values of the caudate and posterior putamen were not significantly different from controls; however, the ADC values in all regions were lower 
in welders than in control subjects. ADC values did not correlate with exposure hours, blood Mn levels or UPDRS3 values within the welder cohort.

\section{DISCUSSION}

While previous studies have focused on the pallidum as the primary target of Mn-induced neurotoxicity, our study demonstrates an increase in the intensity index in all regions of the basal ganglia in Mn-exposed welders. Pallidal hyperintensities in Mn toxicity have been associated with subsequent gliosis and neurodegeneration of the globus pallidus at autopsy. ${ }^{1324}$ Animal and human studies demonstrated Mn deposition throughout the basal ganglia. ${ }^{425}$ Therefore, it is likely that the increased T1 signal in caudate and putamen indexes is also secondary to Mn toxicity in these extra-pallidal regions, although confirmation would require pathological studies in occupationally exposed welders. Clearly, future studies investigating Mn neurotoxicity should include investigation of extra-pallidal basal ganglia regions of interest to understand the broader extent of Mn-induced neurotoxicity.

While the pallidal index has been previously used as the standard imaging marker of welding exposure; the combined basal ganglia, caudate, anterior putamen and posterior putamen intensity indices all showed stronger correlations with exposure hours. Of all the regional indices, the addition of caudate region sampling provided the greatest improvement in the hierarchical regression modelling of exposure hours and was the index most correlated with exposure hours. Alternatively, the combined basal ganglia sampling has the advantage of incorporating data from all four basal ganglia regions, including the traditionally used pallidal index. While the $\mathrm{T} 1$ intensity indices appeared to be correlated with exposure, all the correlations were modest. This could be explained by the fact that welding exposure time is a relatively crude measure of $\mathrm{Mn}$ exposure and does not take into account environmental conditions, such as ventilation or the use of personal protective equipment.

No indices correlated with the UPDRS3 or age, and the caudate index had an inverse relationship with Mn levels in this study. This is likely secondary to the minimal clinical signs and a narrow range of UPDRS3 scores within this welder cohort. Including only asymptomatic healthy workers improves the generalisability of these findings and minimises the risk of accidentally including those with confounding idiopathic Parkinson's disease. However, examining welders with more parkinsonian features will be necessary to truly evaluate if there is a correlation between regional intensity values and clinical signs of parkinsonism as measured by the UPRDS3. Age was controlled for in this study by matching each welding subject to a control subject \pm 4 years. This study has a relatively small sample size for within cohort analysis, and therefore, we did not perform subsequent age adjustments in the statistical analysis. No studies have reported on a change in T1 basal ganglia intensity indices with age; however, overall grey matter T1 intensity has been reported to diminish with age ${ }^{26}$ and hence is unlikely to cause the increased signal identified in Mn exposure. The lack of a consistent association between blood Mn levels and the intensity indices is not surprising since $\mathrm{Mn}$ has a relatively short half-life in blood with high variability ${ }^{27-29}$ but deposition in the pallidum persists for months after exposure. ${ }^{24} \mathrm{In}$ addition, it has recently been reported that Mn may bypass the blood-brain barrier and travel into the central nervous system via the olfactory nerve making blood levels even less reliable. ${ }^{30}$

Whereas the TI intensity indexes were elevated in all four regions, ADC values on DWI were different between welders and controls only in the globus pallidus and anterior putamen. This small but significant restriction in the welder ADC values is interesting as this was a cohort with no symptomatic complaints and only minimal elevation in UPRDS3 
scores. DWI may prove to be a reliable antecedent biomarker of Mn-induced neurotoxicity. There are only two previous reports on DWI/DTI in subjects with Mn exposure for comparison. The first was a single subject with symptomatic Mn toxicity secondary to cirrhosis who demonstrated similar, but more reduced ADC values in the globus pallidus. ${ }^{17}$ The second report focused on disruptions in the white matter connections to the cortex and did not examine the basal ganglia. ${ }^{31}$

DWI studies comparing other idiopathic parkinsonian syndromes including idiopathic Parkinson's disease, progressive supranuclear palsy and multiple system atrophy found no differences between the ADC in basal ganglia regions of subjects with clinical parkinsonism diagnosed with these syndromes and healthy controls. ${ }^{32}$ However, Favrole et a ${ }^{33}$ have demonstrated interesting parallel findings in Wilson's disease. Wilson's disease is a disorder of copper metabolism resulting in extrapyramidal and parkinsonian symptoms secondary to metal deposition within the basal ganglia. Brain copper deposition in Wilson's disease produces similar T1 pallidal hyperintensities to those associated with Mn exposure. In Favrole's study, subjects with presymptomatic Wilson's disease had a restricted diffusion pattern in the putamen, whereas patients with clinically symptomatic Wilson's disease demonstrated increased putamen ADC values. Favrole et $a \beta^{3}$ hypothesised that this may represent initial gliosis or inflammation with secondary cytotoxic edema proceeding the degeneration and cell death usually seen at autopsy. Similarly, we speculate that preclinical or early Mn toxicity may be associated with restrictive ADC pattern in the basal ganglia that later evolves into an elevation of the ADC with the development of obvious clinical symptoms and more severe neuropathology. Further studies in symptomatic welders would be required to test this hypothesis.

Pathophysiologic interpretation of this study is limited by the lack of data on the potential effect of a deposited paramagnetic metal like $\mathrm{Mn}$ in brain tissue on DWI imaging. However, in a previous PET imaging study with $6-\left[{ }^{18}\right.$ F]fluoro-L-DOPA (FDOPA) using asymptomatic welders, we found a consistent reduction in FDOPA uptake in the caudate despite the absence of clinical parkinsonism indicating preclinical dysfunction in the nigrostriatal dopamine system. ${ }^{14}$ This, in conjunction with similar findings in Wilson's disease, suggests that these DWI abnormalities represent underlying pathology and are not just an artefact related to Mn deposition. In addition, with two studies now demonstrating evidence of early basal ganglia dysfunction using complementary but distinct functional imaging modalities, these data suggest that there is a period of preclinical neurotoxicity in humans with occupational Mn exposure. ADC values may differ between subjects older and younger than 60 years. However, ADC values do not appear to vary in adult subjects aged $<60$ years. ${ }^{34}$ As our subjects were all $<60$ years old, we did not adjust for age when reporting these statistics. In addition, age over 60 years was associated with increases in ADC values so it is unlikely that the lower ADC values in welders can be explained by age when compared to a match control cohort. Of course, further research will be required to understand these functional changes and to establish a threshold for Mn neurotoxicity in the basal ganglia.

Welding fumes also contain a number of elements and gases, ${ }^{35}$ and this study cannot exclude a contribution from other substances in welding fumes. However, Mn has been historically associated with parkinsonism, ${ }^{36}$ and these welders had both elevated blood Mn levels and the characteristic increased pallidal signal clearly documenting their metal exposure. ${ }^{8}$ Moreover, this study demonstrates an association between common types of welding exposures and imaging characteristics but does not address causation or evaluate the effects of other metals known to be present in welding fumes. A substantially larger population of exposed workers will be needed to demonstrate dose-responsive DWI and longitudinal changes in the basal ganglia. Welding exposure time as calculated above also does not account for work conditions or personal protective equipment. Unfortunately, we 
have limited site-specific or individualised data on the use of equipment such as respirators for this cohort, given the lack of required use or documentation of use by employers in the USA. Furthermore, eventual imaging-pathological correlations will be required to understand the meaning of these imaging findings.

In conclusion, welders demonstrated elevated basal ganglia indices consistent with diffuse Mn deposition. The combined basal ganglia, caudate and putamen indices were better correlated with exposure than the traditionally used pallidal index, and more inclusive sampling of basal ganglia regions appears to result in better markers of exposures. Elevated indices were also associated with DW abnormalities in the globus pallidus and anterior putamen, suggesting that the increased signal in these regions is associated with neuronal damage.

\section{Acknowledgments}

Funding This study was sponsored by the following: Michael J. Fox Foundation, NIH grants: R01 ES013743, K24 ES017765, KL2 RR024994, P42ES04696, 5T32NS007205-27, RO1 NS41509, RO1 NS058714, the Clinical Science Translational Award NCRR UL1 RR024992, the Neuroscience Blueprint grant NS057105, the American Parkinson's Disease Association (APDA) Advanced Research Center at Washington University, the Greater St. Louis Chapter of the APDA, the McDonnell Center for Higher Brain Function and the Barnes-Jewish Hospital Foundation (Elliot Stein Family Fund and Jack Buck Fund for PD Research).

Competing interests The authors of this paper have received research support from the American Parkinson's Disease Association, Merck, Chiltern, TEVA, Eisai, Medivation, Neurogen, Huntington Disease Society of American, the Michael J. Fox Foundation, McDonnell Center, Bander Foundation, the Barnes Jewish Hospital Foundation, NIH and the Department of Defense.

\section{REFERENCES}

1. Meeker JD, Susi P, Flynn MR. Manganese and welding fume exposure and control in construction. J Occup Environ Hyg. 2007; 12:943-951. [PubMed: 17963139]

2. Racette BA, McGee-Minnich L, Moerlein SM, et al. Welding-related parkinsonism: clinical features, treatment, and pathophysiology. Neurology. 2001; 56:8-13. [PubMed: 11148228]

3. Simon, RP.; Greenberg, DA.; Aminoff, MJ. Movement disorders. In: Simon, RP.; Greenberg, DA.; Aminoff, MJ., editors. Clinical Neurology. 7th edn.. McGraw-Hill; 2009. http:// www.accessmedicine.com/content.aspx?aID=5149365

4. Eriksson H, Magiste K, Plantin LO, et al. Effects of manganese oxide on monkeys as revealed by a combined neurochemical, histological and neurophysiological evaluation. Arch Toxicol. 1987; 61:46-52. [PubMed: 3439874]

5. Tapin D, Kennedy G, Lambert J, et al. Bioaccumulation and locomotor effects of manganese sulfate in Sprague-Dawley rats following subchronic (90 days) inhalation exposure. Toxicol Appl Pharmacol. 2006; 211:166-174. [PubMed: 16112697]

6. Dorman DC, Struve MF, Marshall MW, et al. Tissue manganese concentrations in young male rhesus monkeys following subchronic manganese sulfate inhalation. Toxicol Sci. 2006; 92:201210. [PubMed: 16624849]

7. Nelson K, Golnick J, Korn T, et al. Manganese encephalopathy: utility of early magnetic resonance imaging. Br J Ind Med. 1993; 50:510-513. [PubMed: 8329316]

8. Kim Y, Kim KS, Yang JS, et al. Increase in signal intensities on T1-weighted magnetic resonance images in asymptomatic manganese-exposed workers. Neurotoxicology. 1999; 20:901-907. [PubMed: 10693971]

9. Dietz MC, Ihrig A, Wrazidlo W, et al. Results of magnetic resonance imaging in long-term manganese dioxide-exposed workers. Environ Res. 2001; 85:37-40. [PubMed: 11161650]

10. Chang Y, Woo ST, Kim Y, et al. Pallidal index measured with three-dimensional T1-weighted gradient echo sequence is a good predictor of manganese exposure in welders. J Magn Reson Imaging. 2010; 31:1020-1026. [PubMed: 20373449] 
11. Fernandez-Rodriguez R, Contreras A, De Villoria JG, et al. Acquired hepatocerebral degeneration: clinical characteristics and MRI findings. Eur J Neurol. 2010; 17:1463-1470. [PubMed: 20491897]

12. Burkhard PR, Delavelle J, Du PR, et al. Chronic parkinsonism associated with cirrhosis: a distinct subset of acquired hepatocerebral degeneration. Arch Neurol. 2003; 60:521-528. [PubMed: 12707065]

13. Pal PK, Samii A, Calne DB. Manganese neurotoxicity: a review of clinical features, imaging and pathology. Neurotoxicology. 1999; 20:227-238. [PubMed: 10385886]

14. Criswell SR, Perlmutter JS, Videen TO, et al. Reduced uptake of [18F]FDOPA PET in asymptomatic welders with occupational manganese exposure. Neurology. 2011; 76:1296-1301. [PubMed: 21471467]

15. Le BD, Mangin JF, Poupon C, et al. Diffusion tensor imaging: concepts and applications. J Magn Reson Imaging. 2001; 13:534-546. [PubMed: 11276097]

16. Baird AE, Warach S. Magnetic resonance imaging of acute stroke. J Cereb Blood Flow Metab. 1998; 18:583-609. [PubMed: 9626183]

17. McKinney AM, Filice RW, Teksam M, et al. Diffusion abnormalities of the globi pallidi in manganese neurotoxicity. Neuroradiology. 2004; 46:291-295. [PubMed: 15045494]

18. Fahn, S.; Elton, RL. Members of the UPDRS Development Committee. Unified Parkinson's disease rating scale. In: Fahn, S.; Marsden, CD.; Goldstein, M., et al., editors. Recent Developments in Parkinson's Disease. New York: Macmillan; 1987. p. 153-163.

19. Hobson AJ, Sterling DA, Emo B, et al. Validity and reliability of an occupational exposure questionnaire for parkinsonism in welders. J Occup Environ Hyg. 2009; 6:324-331. [PubMed: 19288335]

20. Fitsanakis VA, Zhang N, Anderson JG, et al. Measuring brain manganese and iron accumulation in rats following 14 weeks of low-dose manganese treatment using atomic absorption spectroscopy and magnetic resonance imaging. Toxicol Sci. 2008; 103:116-124. [PubMed: 18234737]

21. Spahr L, Butterworth RF, Fontaine S, et al. Increased blood manganese in cirrhotic patients: relationship to pallidal magnetic resonance signal hyperintensity and neurological symptoms. Hepatology. 1996; 24:1116-1120. [PubMed: 8903385]

22. Basser PJ, Mattiello J, LeBihan D. Estimation of the effective self-diffusion tensor from the NMR spin echo. J Magn Reson B. 1994; 103:247-254. [PubMed: 8019776]

23. Shimony JS, Sheline YI, D’Angelo G, et al. Diffuse microstructural abnormalities of normalappearing white matter in late life depression: a diffusion tensor imaging study. Biol Psychiatry. 2009; 66:245-252. [PubMed: 19375071]

24. Newland MC. Animal models of manganese's neurotoxicity. Neurotoxicology. 1999; 20:415-432. [PubMed: 10385901]

25. Larsen NA, Pakkenberg H, Damsgaard E, et al. Topographical distribution of arsenic, manganese, and selenium in the normal human brain. J Neurol Sci. 1979; 42:407-416. [PubMed: 512674]

26. Salat DH, Lee SY, van der Kouwe AJ, et al. Age-associated alterations in cortical gray and white matter signal intensity and gray to white matter contrast. Neuroimage. 2009; 48:21-28. [PubMed: 19580876]

27. Zheng W, Kim H, Zhao Q. Comparative toxicokinetics of manganese chloride and methylcyclopentadienyl manganese tricarbonyl (MMT) in Sprague-Dawley rats. Toxicol Sci. 2000; 54:295-301. [PubMed: 10774811]

28. Aschner M, Guilarte TR, Schneider JS, et al. Manganese: recent advances in understanding its transport and neurotoxicity. Toxicol Appl Pharmacol. 2007; 221:131-147. [PubMed: 17466353]

29. Apostoli P, Lucchini R, Alessio L. Are current biomarkers suitable for the assessment of manganese exposure in individual workers? Am J Ind Med. 2000; 37:283-290. [PubMed: 10642418]

30. Sen S, Flynn MR, Du G, et al. Manganese accumulation in the olfactory bulbs and other brain regions of "asymptomatic" welders. Toxicol Sci. 2011; 121:160-167. [PubMed: 21307282]

31. Kim Y, Jeong KS, Song HJ, et al. Altered white matter microstructural integrity revealed by voxelwise analysis of diffusion tensor imaging in welders with manganese exposure. Neurotoxicology. 2010; 32:100-109. [PubMed: 21111757] 
32. Paviour DC, Thornton JS, Lees AJ, et al. Diffusion-weighted magnetic resonance imaging differentiates Parkinsonian variant of multiple-system atrophy from progressive supranuclear palsy. Mov Disord. 2007; 22:68-74. [PubMed: 17089396]

33. Favrole $\mathrm{P}$, Chabriat $\mathrm{H}$, Guichard JP, et al. Clinical correlates of cerebral water diffusion in Wilson disease. Neurology. 2006; 66:384-389. [PubMed: 16476937]

34. Naganawa S, Sato K, Katagiri T, et al. Regional ADC values of the normal brain: differences due to age, gender, and laterality. Eur Radiol. 2002; 12(Suppl 3):S114-S117. [PubMed: 12522618]

35. NIOSH. , editor. Criteria for a Recommended Standard: Occupational Exposure to Welding, Brazing, and Thermal Cutting. Cincinnati, OH: U.S. Department of Health and Human Services, Public Health Service, Centers for Disease Control, National Institute for Occupational Safety and Health, DHHS (NIOSH); 1988. Publication No. 88_110.

36. Rodier J. Manganese poisoning in Moroccan miners. Br J Ind Med. 1955; 12:21-35. [PubMed: 14351643] 


\section{What is known about this subject}

Occupational manganese exposure leads to cerebral deposition of the metal, which can be measured on T1-weighted MRI imaging. Currently, the T1 signal intensity in the globus pallidus is the standard marker of manganese exposure despite being only loosely correlated with exposure and having no clear association with neurotoxicity. 


\section{What this paper adds}

This paper demonstrates that manganese deposits more diffusely in the human brain than previously recognised as increased $\mathrm{T} 1$ signal is present throughout the basal ganglia. Combining multiregion measurements of the $\mathrm{T} 1$ signal provides a better marker of cumulative manganese exposure, and associated diffusion weighted imaging may provide a marker of neurotoxicity. 


\section{Policy implications}

These findings have important practical implications as improved in vivo measures of manganese exposure and neurotoxicity are essential in setting acceptable exposure thresholds. 
A

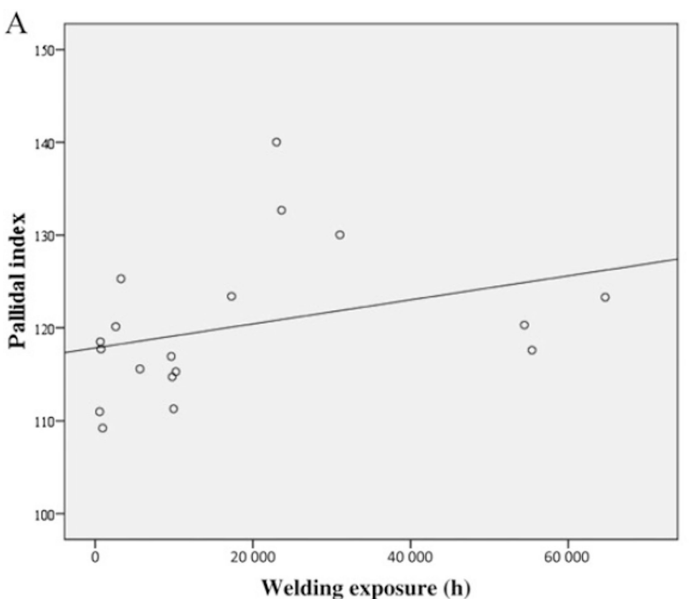

C
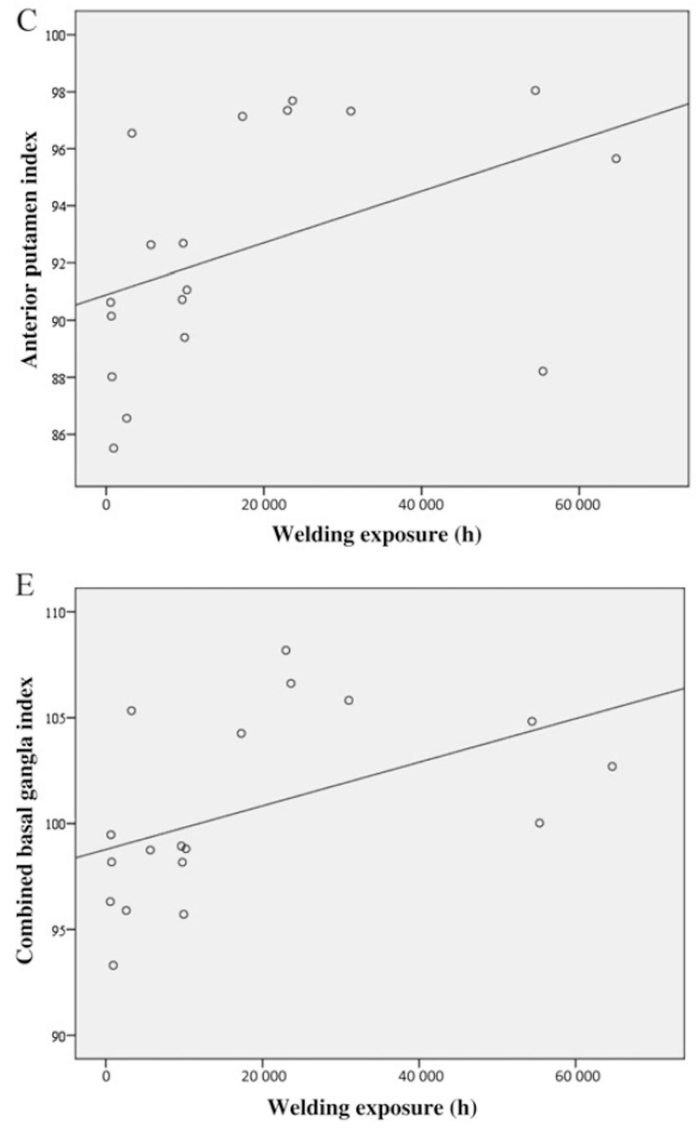

$\mathrm{B}$

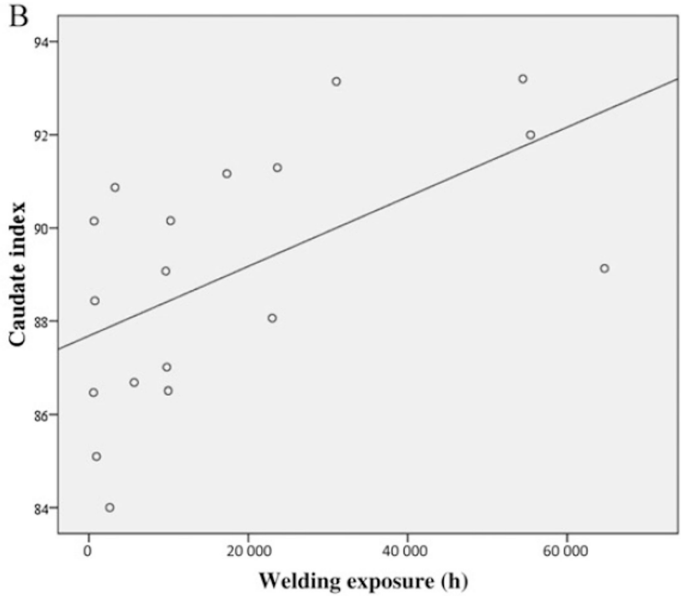

$\mathrm{D}$

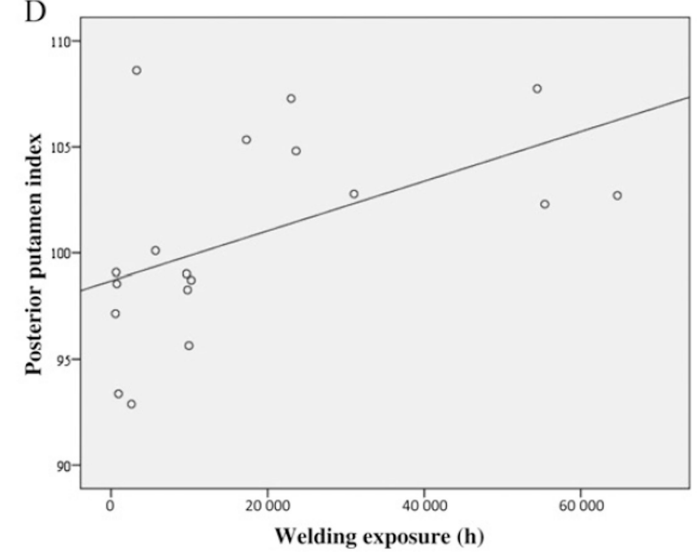

Figure 1.

Correlations between regional intensity indices and cumulative exposure hours. Scatter plots demonstrate the correlations between the globus pallidus (A), caudate (B), anterior putamen (C), posterior putamen (D), combined basal ganglia (E) indices and cumulative exposure hours. 
Table 1

Demographic data by diagnostic category

\begin{tabular}{llll}
\hline & Normal control $(\mathbf{n = 1 8})$ & Welders $(\mathbf{n = 1 8})$ & p Value \\
\hline Gender (female/male) & $1 / 17$ & $1 / 17$ & \\
Age, median in years (IQR) & $37.5(25.7-52.0)$ & $40.5(29.2-50.2)$ & $0.558^{*}$ \\
UPDRS3 score, median (IQR) & $0(0-3)$ & $6.25(2.75-10.25)$ & \multirow{\$}{\Delta}{$0.001^{*}$}
\end{tabular}

Mann-Whitney U test compared welders with normal controls.

UPDRS3, Unified Parkinson's Disease Rating Scale motor subsection 3. 
Table 2

$\mathrm{T} 1$ intensity indices in welders and normal controls

\begin{tabular}{lccc}
\hline & Normal control $(\mathbf{n}=18)$ & Welders $(\mathbf{n}=18)$ & p Value \\
\hline Pallidal index, median (IQR) & $112.35(109.59-116.16)$ & $118.11(115.14-123.87)$ & $0.002^{*}$ \\
Caudate index, median (IQR) & $86.7(85.0-88.44)$ & $89.10(86.64-91.20)$ & $0.019^{*}$ \\
Anterior putamen index, median (IQR) & $88.58(86.69-90.12)$ & $91.85(89.10-97.18)$ & $0.005^{*}$ \\
Posterior putamen index, median (IQR) & $96.66(93.99-98.22)$ & $99.59(97.99-104.94)$ & $0.002^{*}$ \\
Basal ganglia index, median (IQR) & $96.55(93.97-98.10)$ & $99.2(97.70-104.95)$ & $0.002^{*}$ \\
\hline
\end{tabular}

* Mann-Whitney U tests compared welders to normal controls. 


\section{Table 4}

ADC measurements in welders and normal controls

\begin{tabular}{llll}
\hline & Normal control $(\mathbf{n}=18)$ & Welders $(\mathbf{n}=18)$ & p Value $^{*}$ \\
\hline Globus pallidus, median ADC ${ }^{\dagger}(\mathrm{IQR})$ & $0.7202(0.6798-0.7576)$ & $0.6844(0.6452-0.7191)$ & $0.03^{\dagger}$ \\
Caudate, median ADC (IQR) & $0.7216(0.7130-0.7356)$ & $0.7176(0.7049-0.7406)$ & 0.29 \\
Anterior putamen, median ADC (IQR) & $0.7041(0.6977-0.7138)$ & $0.6862(0.6788-0.7000)$ & $0.004^{\dagger}$ \\
Posterior putamen, median ADC (IQR) & $0.7258(0.7091-0.7333)$ & $0.7088(0.6821-0.7295)$ & 0.24 \\
\hline
\end{tabular}

* Mann-Whitney U test compared welders to normal controls.

${ }^{\dagger}$ ADC values are $\times 10^{-3} \mathrm{~mm}^{2} / \mathrm{s}$.

${ }^{\star}$ Indicates significance at the p $₫ 0.05$ level.

ADC, apparent diffusion coefficient. 